\title{
Two-photon exchange and elastic scattering of positrons/electrons on the proton
}

\section{M. Nikolenko, ${ }^{a}$ J. Arrington, ${ }^{b}$ L. M. Barkov, ${ }^{a}$ V. F. Dmitriev,${ }^{a, c}$ V. V. Gauzshteyn, ${ }^{d}$} R. A. Golovin, ${ }^{a, c}$ A. V. Gramolin, ${ }^{a}$ R. J. Holt, ${ }^{b}$ V. V. Kaminsky, ${ }^{a}$ B. A. Lazarenko, ${ }^{a}$ S. I. Mishnev, ${ }^{a}$ N. Yu. Muchnoi, ${ }^{a}, c$ V. V. Neufeld, ${ }^{a}$ A. V. Osipov ${ }^{\dagger}{ }^{d}$ I. A. Rachek, ${ }^{a}$ R. Sh. Sadykov, ${ }^{a}$ Yu. V. Shestakov, ${ }^{a, c}$ V. N. Stibunov, ${ }^{d}$ D. K. Toporkov, ${ }^{a, c}$ H. de Vries, ${ }^{e}$ S. A. Zevakov ${ }^{a}$ and V. N. Zhilich ${ }^{a}$

${ }^{a}$ Budker Institute of Nuclear Physics, Novosibirsk, Russia

${ }^{b}$ Argonne National Laboratory, Argonne, USA

${ }^{c}$ Novosibirsk State University, Novosibirsk, Russia

${ }^{d}$ Nuclear Physics Institute of Tomsk Polytechnic University, Tomsk, Russia

${ }^{e}$ NIKHEF, Amsterdam, The Netherlands

E-mail: D.M.Nikolenko@inp.nsk.su

\begin{abstract}
We report the experiment on a precise comparison of $\left(e^{+} p\right)$ and $\left(e^{-} p\right)$ elastic scattering cross sections, which gives a direct experimental evidence for the two-photon exchange contribution to this reaction. Such data are in demand now, because they, most likely, may explain the dramatic disagreement of proton electromagnetic form factors measurements in the polarization transfer experiments at TJNAF with previous unpolarized measurements using a Rosenbluth separation technique. Experiment was performed recently at VEPP-3 storage ring at the energy of positron/electron beams of $1.6 \mathrm{GeV}$ and at $e^{+} / e^{-}$scattering angles around $20^{\circ}$ and $65^{\circ}$. The preliminary results for the cross sections ratio $R=\sigma\left(e^{+} p\right) / \sigma\left(e^{-} p\right)$ are presented.
\end{abstract}

35th International Conference of High Energy Physics

July 22-28, 2010

Paris, France

\footnotetext{
* Speaker.

$\dagger$ Deceased.
} 
Elastic electromagnetic form factors of the proton are important characteristics of this particle [1]. The proton's electric $G_{E}\left(Q^{2}\right)$ and magnetic $G_{M}\left(Q^{2}\right)$ form factors describe the distribution of charge and current inside the proton as functions of four-momentum transfer squared $Q^{2}$. Until recently these form factors were determined only by an analysis of differential cross section of the elastic (ep)-scattering. Such a procedure is called Rosenbluth separation (RS) because it is based on the Rosenbluth formula [2] describing the elastic $(e p)$-scattering cross section in one-photon exchange approximation. RS method requires measurements of the unpolarized elastic cross section at fixed $Q^{2}$, but with different electron scattering angles and incident beam energies (or, equivalently, with different values of $\varepsilon$, virtual photon polarization [1]).

In the mid-nineties, it became possible to use polarization transfer (PT) method to study nucleon electromagnetic form factors. This method was originally proposed back in 1970's [3]. In the PT method a polarization of the recoil proton in the process of elastic scattering of longitudinally polarized electrons on an unpolarized hydrogen target is measured. In such a case the ratio $G_{E} / G_{M}$ is directly proportional to the ratio of transverse and longitudinal polarizations of recoil protons.

Measurements performed in TJNAF with PT method [4] gave the results that are in contradiction with the results obtained by RS. This is clearly seen in Fig. 1, where the polarized data of TJNAF as well as several results of RS measurements are shown. The latter include global analysis by Walker [5] and new accurate RS measurements of TJNAF [6]. Note that solid lines are the quadratic fits for these two groups of data.

It is often argued that the origin of these discrepancies is the failure of the one-photon approximation to precisely describe the results of RS experiments. However, the consideration of the two-photon exchange (TPE) contribution encounters difficulties, both theoretical and experimental character. The problem has caused the appearance of many new theoretical studies performed in the various approaches as well as the suggestions of new experiments for the determination of TPE contribution to this reaction. The most direct way to determinate TPE contribution is a measurement of the positron-proton and electron-proton scattering cross sections ratio $R=\sigma\left(e^{+} p\right) / \sigma\left(e^{-} p\right)$.

Here we report the experiment on a precise measurement of $R$, which was performed at the storage ring VEPP-3 (Novosibirsk) with positron/electron beams energy of $1.6 \mathrm{GeV}$ [7]. Elastic scattering events were detected simultaneously in the three angular ranges: small angle (SA), $\theta_{e} \approx$ $10^{\circ}$; middle angle (MA), $\theta_{e} \approx 20^{\circ}$; and large angle (LA), $\theta_{e} \approx 65^{\circ}$. Corresponding $\varepsilon$ values were $\approx 0.98,0.94,0.46$ and values of $Q^{2}$ were $\approx 0.08,0.28,1.5 \mathrm{GeV}^{2}$. The SA scattering was used for luminosity monitoring because in this case TPE contribution should be insignificant, since $\varepsilon$ is close to 1 and $Q^{2}$ is small.

A typical working cycle consisted in: i) the storing of $e^{+} / e^{-}$beams up to $40 \div 50 \mathrm{~mA}$; ii) ramping beam energy (from 0.35 to $1.6 \mathrm{GeV}$ ); iii) switching on the internal gas target and then data taking; iv) returning to the injection energy. The cycles of $e^{+} / e^{-}$beams were alternated regularly. The full cycle with two beams $\left(e^{+}\right.$and $\left.e^{-}\right)$takes 80 minutes, of which $2 \times 22$ minutes was spent on the data taking, 22 or 8 minutes on the storage of $e^{+}$or $e^{-}$beams and energy ramping. We have performed about 1100 such double cycles (runs).

Internal gas target is based on an open end storage cell, having an elliptical cross-section $13 \times 24 \mathrm{~mm}^{2}$ and a length of $400 \mathrm{~mm}$; the cell is cooled to $\approx 20 \mathrm{~K}$. Hydrogen flux is injected to the cell center for providing a target thickness of $\approx 10^{15}$ at. $/ \mathrm{cm}^{2}$.

The detector (Fig. 2) is comprised of two nearly identical parts placed symmetrically with 


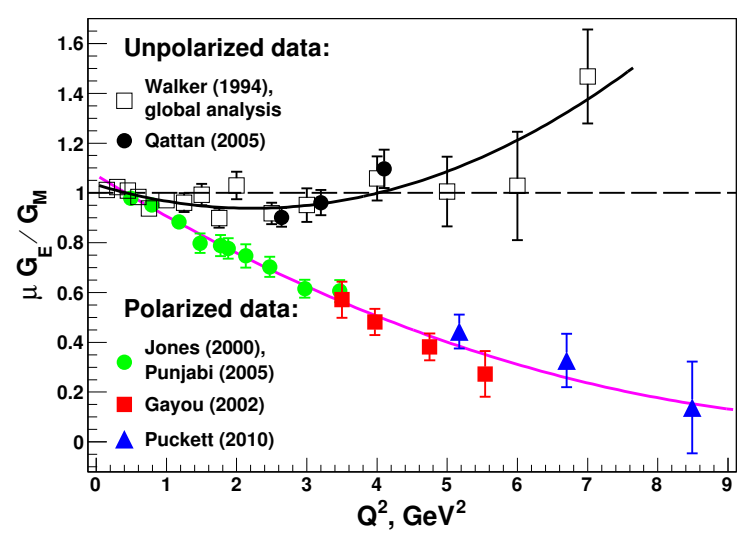

Figure 1: Comparison of polarized [4] and unpolarized $[5,6]$ data for the ratio $\mu G_{E} / G_{M}$.

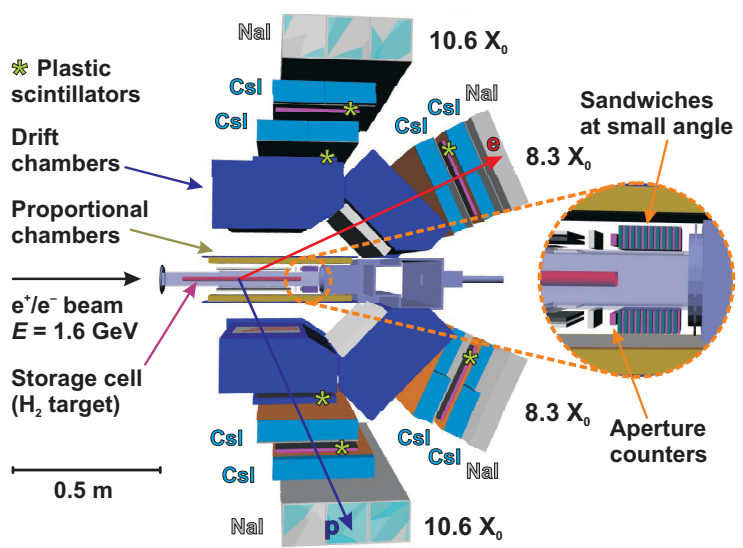

Figure 2: Schematic side view of the particle detection system.

respect to the storage ring median plane. Azimuthal angle acceptance of each of two systems is $60^{\circ}$, so the total acceptance is $\Delta \phi=120^{\circ}$. Scattered positron/electron and recoil proton are detected in coincidence, which facilitates the separation of the elastic scattering events from the various background events by using kinematic correlations.

To select the events of $(e p)$-scattering we used the parameters cuts (in different combination for SA, MA or LA scattering) connected with following main sources: i) correlation between polar angles of $e^{+}$or $e^{-}$and $p$; ii) correlation between azimuthal angles of $e^{+}$or $e^{-}$and $p$; iii) correlation between positron or electron scattering angle and proton energy; iv) correlation between positron or electron scattering angle and its energy; v) $\Delta E-E$ analysis for proton identification; vi) time-offlight analysis for the identification of proton with low energy.

For the suppressing of the systematic errors it was important to keep the identical conditions during data taking with positron and electron beams. Special attention should be paid to equality of energies, currents and positions of the beams, equality of target thickness, accelerator related background, stability of detector efficiency.

Positron/electron beams energies were measured regularly and with good accuracy during data taking by Compton backscattering setup. We had three sources of information on the $e^{+} / e^{-}$ beams positions: VEPP-3 beam position monitors, moveable beam scrapers and vertex position reconstruction using coordinate system of the detector. Summation of information from these three sources makes it possible to determine the position of beams with an accuracy of $0.1 \mathrm{~mm}$.

Fig. 3 shows raw ratio $R$ for LA as a function of run's number (in other words, variation of $R$ during the experiment). Preliminary results for $R$ (obtained for LA and MA) after normalizing to SA and applying radiative corrections are shown in Fig. 4 together with the previous measurements [8]. Error bars in this figure correspond to statistical uncertainty, systematic uncertainty is estimated as $3 \cdot 10^{-3}$. One can see, that new measurements have a significantly higher accuracy. It is clear, that contribution of radiative corrections to ratio $R$ is considerable, therefore its careful accounting is required. At the current phase of data analysis we have used a simple soft photon approximation described, for example, in [9]. Now we are realizing a more sophisticated approach to accounting for radiative correction. This approach includes a development of $(e p)$-scattering events generator, based on the calculation of bremsstrahlung cross section beyond the soft photon approximation [10], and Monte-Carlo simulation of detector response using Geant4 toolkit. 


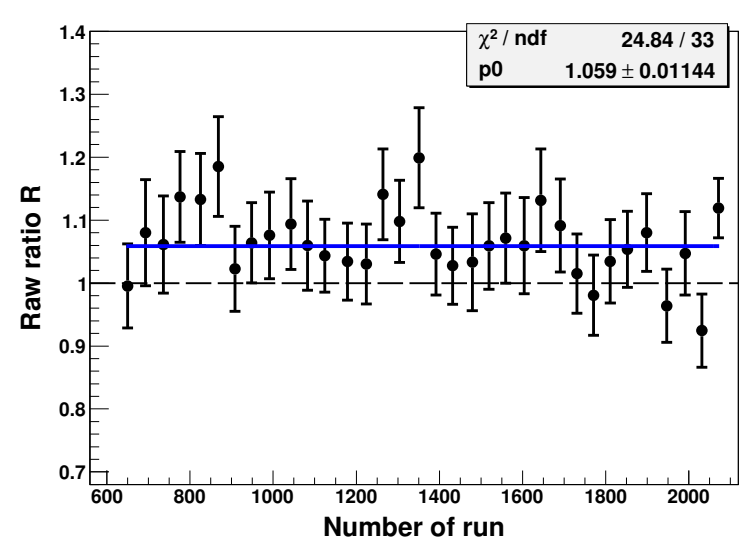

Figure 3: Raw data for the ratio $R$ as a function of run's number (for scattering at LA). The solid line shows the average value $(R=1.059 \pm 0.011)$.

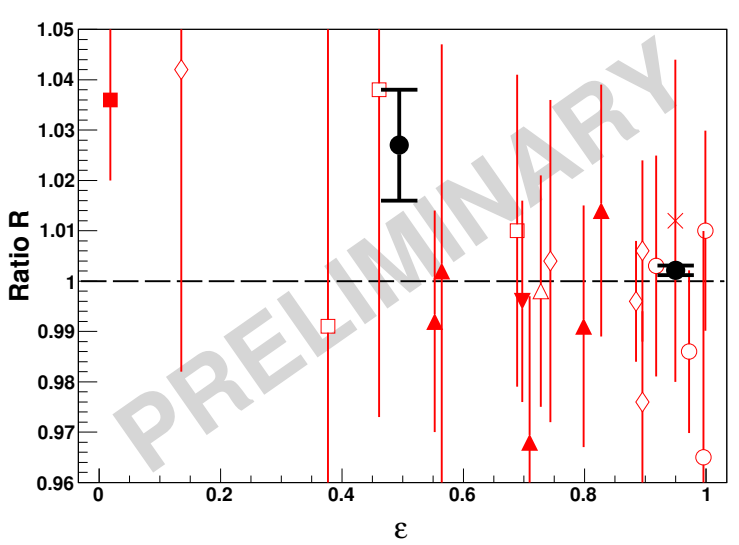

Figure 4: Comparison of preliminary results for the ratio $R$ with the previous measurements [8] (for $Q^{2}<2 \mathrm{GeV}^{2}$ ): $\diamond$ Yount (1962); $\square$ Browman, run 1 (1965); $\triangle$ Browman, run 2 (1965); $\nabla$ Anderson (1966); × Bartel (1967); $\boldsymbol{\square}$ Bouquet (1968); $\boldsymbol{\Delta}$ Anderson (1968); O Mar (1968); • this experiment.

\section{Acknowledgments}

We acknowledge the staff of VEPP-3 for excellent performance of the ring during the data collection runs. We are grateful to V. S. Fadin and A. L. Feldman for useful discussions. This work was supported in part by Russian Foundation for Basic Research under grants 08-02-00624-a, 08-02-01155-a, 10-02-08433-z; by Federal Agency for Education under State Contract P522; by Federal Agency for Science and Innovations under Contract 02.740.11.0245.1; by US DOE under grant DE-AC02-06CH11357; and by US NSF under grant PHY-03-54871.

\section{References}

[1] C.E. Hyde-Wright and K. de Jager, Annu. Rev. Nucl. Part. Sci. 54 (2004) 217; C.F. Perdrisat, V. Punjabi and M. Vanderhaeghen, Prog. Part. Nucl. Phys. 59 (2007) 694.

[2] M.N. Rosenbluth, Phys. Rev. 79 (1950) 615.

[3] A.I. Akhiezer and M.P. Rekalo, Sov. J. Part. Nucl. 4 (1974) 277;

R.G. Arnold, C.E. Carlson and F. Gross, Phys. Rev. C 23 (1981) 363.

[4] M.K. Jones, et al., Phys. Rev. Lett. 84 (2000) 1398; V. Punjabi, et al., Phys. Rev. C 71 (2005) 055202; O. Gayou, et al., Phys. Rev. Lett. 88 (2002) 092301; A.J.R. Puckett, et al., Phys. Rev. Lett. 104 (2010) 242301.

[5] R.C. Walker, et al., Phys. Rev. D 49 (1994) 5671.

[6] I.A. Qattan, et al., Phys. Rev. Lett. 94 (2005) 142301.

[7] J. Arrington, et al., nucl-ex/0408020.

[8] D. Yount and J. Pine, Phys. Rev. 128 (1962) 1842; A. Browman, F. Liu, and C. Schaerf, Phys. Rev. 139 (1965) B1079; R.L. Anderson, et al., Phys. Rev. Lett. 17 (1966) 407; W. Bartel, et al., Phys. Lett. B 25 (1967) 242; B. Bouquet, et al., Phys. Lett. B 26 (1968) 178; R.L. Anderson, et al., Phys. Rev. 166 (1968) 1336; J. Mar, et al., Phys. Rev. Lett. 21 (1968) 482.

[9] R. Ent, et al., Phys. Rev. C 64 (2001) 054610.

[10] V.S. Fadin and A.L. Feldman, private communication. 